\title{
Automatic identification of wheat fungi diseases using a convolutional neural network
}

\author{
Genaev M.A. ${ }^{1,2,3 *}$, Skolotneva E.S. ${ }^{1,2}$, Gultyaeva E.I. ${ }^{4}$, Orlova E.A. ${ }^{5}$, Bekhtold N.P. ${ }^{5}$, \\ Afonnikov D.A. ${ }^{1,2,3}$ \\ ${ }^{1}$ Institute of Cytology and Genetics of Siberian Branch of the Russian Academy of Sciences, Novosibirsk, \\ Russia \\ ${ }^{2}$ Novosibirsk State University, Novosibirsk, Russia \\ ${ }^{3}$ Kurchatov Genomic Center of the Institute of Cytology and Genetics of Siberian Branch of the Russian \\ Academy of Sciences, Novosibirsk, Russia \\ ${ }^{4}$ All-Russian Institute of Plant Protection, St. Petersburg-Pushkin, Russia \\ ${ }^{5}$ Siberian Research Institute of Plant Production and Breeding - Branch of the Institute of Cytology and \\ Genetics of Siberian Branch of the Russian Academy of Sciences, Novosibirsk, Russia \\ *email:mag@bionet.nsc.ru
}

Diseases of cereals caused by pathogenic fungi can significantly reduce crop yields. Many cultures are exposed to them. The disease is difficult to control on a large scale, therefore, one of the relevant approaches is crop monitoring, which helps to identify the disease at an early stage and take measures to prevent its spread. One of the effective methods of control are approaches based on the identification of the disease from digital images that can be obtained by a mobile phone camera. In this paper, we present a dataset of 2414 wheat images, which represent 5 different diseases: leaf rust, powder mildew, septoria, stem rust, yellow rust. To predict the disease at early stages of development, we additionally annotated the image with the seedlings tag, if the plant corresponds to an early stage of development. Based on this dataset, we trained a model that, from a digital image of wheat plants, determines the stage of development and the type of disease. The algorithm is based on the CNN neural network of the EfficientNet-B0 architecture. The resulting model demonstrates an accuracy of 94.2\%. The WFD (wheat fungi diseases) dataset used for training is available for public access at http://wfd.sysbio.ru/. The proposed recognition model was implemented for use in the form of the @wheat_healthy_bot bot in the Telegram messenger https://t.me/wheat_healthy_bot.

Acknowledgements: Development of algorithms for wheat fungi diseases identification were funded by the Kurchatov Genomic Center of the Institute of Cytology and Genetics, SB RAS, agreement with the Ministry of Education and Science of the Russian Federation No. 075-15-2019-1662. 